\title{
Artificial Chiral Media Using Conical-Coil Wire Inclusions
}

\author{
Guy DeMartinis ${ }^{1}$ and Dikshitulu Kalluri ${ }^{2}$
}

\author{
${ }^{1}$ Submillimeter-Wave Technology Laboratory, University of Massachusetts Lowell, Lowell, Massachusetts, USA \\ ${ }^{2}$ Department of Electrical and Computer Engineering, University of Massachusetts Lowell, Lowell, Massachusetts, USA \\ *corresponding author, E-mail: guy_demartinis@uml.edu
}

\begin{abstract}
The electromagnetic response of the electrically small conical wire coil as a chiral inclusion is described. An existing model of the helical coil wire inclusion is extended to model the conical coil wire inclusion, using the Method of Moments (MoM) to determine the dominant resonant circuit impedance of the inclusion. Material parameters are determined using mixing relations with polarizability coefficients expressed for the conical coil inclusion geometry. The polarization conversion of a dielectric slab loaded with conical coil inclusions is predicted and compared to simulated results using a forward scattering technique.
\end{abstract}

Keywords: Chiral Media; Conical Coils; Electromagnetic Modeling; Helical Inclusion; Method of Moments; Mixing Relations; Tumble Averaged Forward Scattering

\section{Introduction}

$\mathrm{T}$ he modeling and synthesis of complex electromagnetic materials has received a great amount of interest during recent decades [1, 2, 3]. Electromagnetic components and systems have been developed possessing unique properties afforded by the development of structured arrays of planar, or randomly oriented three-dimensional, inclusions $[4,5,6]$. In this document we investigate the behavior of electrically small multi-turn conical coil wire inclusions as a geometric extension of the common multi-turn helical coil wire inclusion [1, 7]. In order to estimate the macroscopic properties of Pasteur media using conical coil inclusions we modify an approach set forth by Bahr and Clausing [7, 8]. The potential for tuning multiple material chiral resonances using the conical coil inclusion geometry is demonstrated via the predicted polarization conversion of a chiral slab in free-space.

The electromagnetic response of randomly oriented helical inclusions within a host medium is that of a Pasteur Medium with the following material constitutive relations [1]:

$$
\begin{aligned}
& \vec{D}=\varepsilon \vec{E}-j \kappa \sqrt{\mu_{h} \varepsilon_{h}} \vec{H} \\
& \vec{B}=\mu \vec{H}+j \kappa \sqrt{\mu_{h} \varepsilon_{h}} \vec{E}
\end{aligned}
$$

The parameter $\kappa$ characterizes the degree of handedness of the material and is called the chirality parameter. The host permittivity is provided by $\varepsilon_{h}$ along with the host permeability as $\mu_{h}$. The effective scalar dielectric permittivity and permeability of the artificial material are given by the symbols $\varepsilon$ and $\mu$ respectively. The average vector fields in the media are given by $\vec{E}, \vec{H}, \vec{D}$ and $\vec{B}$.

\section{Theory: Effective Medium Parameters}

\subsection{Polarizability of conical coil inclusion}

The dimensions of a 3-turn conical coil inclusion are identified in Fig. 1 below. The pitch, $P$, is the spacing between turns of the coil and is held constant in this work; the radius of each coil is identified by $a_{i}$ with the zeroth turn initial radius $a_{0}$ identified in the figure; the conical angle is taken relative to the axis of symmetry of the coil and is labelled as $\theta_{c}$.

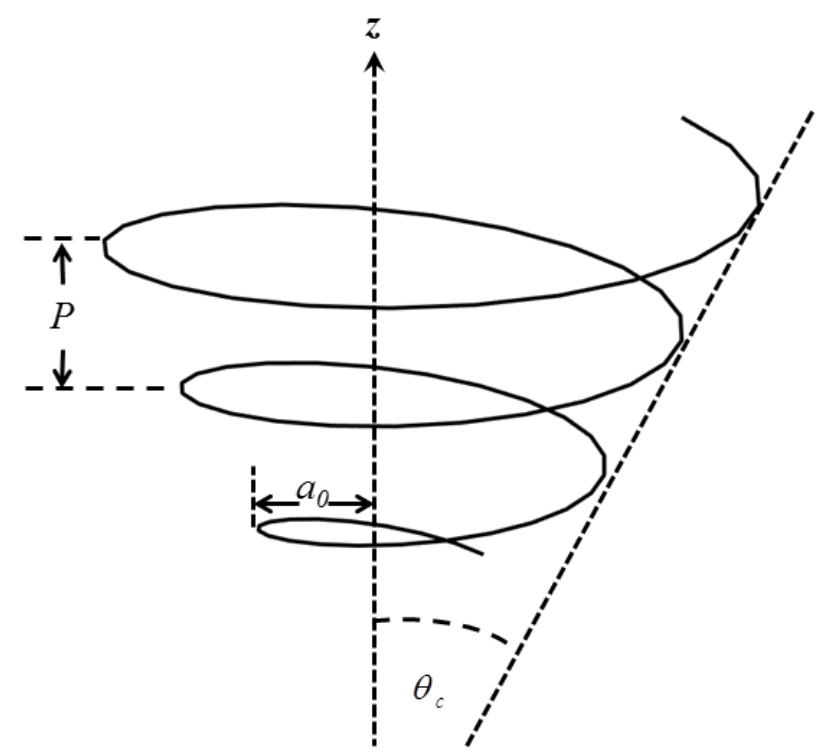

Figure 1: Dimensions of a Right-Handed 3-Turn Conical Coil Inclusion

In order to derive relations for the dipole moments of the conical coil, expressions for the induced emfs for the conical coil geometry must be determined. The induced electromotive force $(e m f)$ arising from the incident electric 
field is given by Faraday's Law:

$$
e m f_{E}=\oint \vec{E} \cdot d \vec{l}
$$

Since the incident electric field is parallel to only that vector component along the coil axis we evaluate this relation to yield:

$$
e m f_{E}= \pm N P E_{0}
$$

where $N$ is the number of turns, $P$ the coil pitch (distance between wires of neighboring turns), $E_{0}$ is the excitation electric field incident upon the coil, and the sign is determined by the handedness of the coil (- left or + right).

Faraday's Law is now expressed as follows for evaluation of this case involving an incident H-Field. Note that we now express the induced emf in terms of integrating the magnetic flux density over the coil cross section:

$e m f_{H}=\oint_{C} \vec{E} \cdot d \vec{l}=-\oint_{S} \frac{\partial \vec{B}}{\partial t} \cdot d \vec{s}$

Assuming a uniform cross section for a multi-turn helical coil of radius $a$, with $H_{0}$ being the excitation magnetic field incident upon the coil, we may evaluate the previous relation to yield:

$$
e m f_{H}=-j \omega N\left(\pi \cdot a^{2}\right) \mu_{h} H_{0}
$$

The emf will increase for a conical coil as the interior cone angle increases since the cross-sectional area intersected by the magnetic flux will increase. We may modify $N$ to provide an estimate for this increased flux linkage by introducing $N^{\prime}$ as defined below. Notice that, in the case of a helical coil, the following relation is equal to the number of turns of the coil but that $N^{\prime}$ becomes larger than $N$ as the cone angle becomes greater than zero.

$N^{\prime}=\sum_{i=0}^{N-1} \frac{A_{i_{a v g}}}{A_{0_{\text {avg }}}}$

where the area $A_{i_{\text {avg }}}$ of the $i^{\text {th }}$ loop is defined as:

$$
A_{i_{\text {avg }}}=\pi \cdot a_{i_{\text {avg }}}^{2}
$$

Although the actual radii of a conical coil changes continuously over the full length of wire we will make use of an approximation and assume a constant-radius-per-turn using the average radius for each full $2 \pi$ coil turn. This approximation allows easy evaluation of estimated impedance contributions in terms of a superposition of elemental loops and dipoles later in this report, but is an estimate of the increase in effective turns $N^{\prime}$. Our evaluation of the emf may be expressed for a conical helix as follows with $a_{0_{a v g}}$ representing the average coil radius for the first (smallest) coil turn:

$e m f_{H} \approx-(j \omega) N^{\prime}\left(\pi \cdot a_{0_{\text {avg }}}^{2}\right) \mu_{h} H_{0}$

From (6)-(9), expressions for the dipole moments may be created both in terms of the electric and magnetic dipole moments as well as in terms of estimates of conical coil characteristic impedance $Z_{C}(10)$ [8]. In [7] the $p_{\text {mez }}$ term is arrived at by modeling the helical coil as a flat disc with the radius of the coil, for the conical coil this term is approximated in a similar manner by using the mean conical coil radius as $a_{\text {mean }}$.

$\left[\begin{array}{ll}p_{\text {eez }} & p_{\text {emz }} \\ p_{\text {mez }} & p_{\text {mmz }}\end{array}\right]=\left[\begin{array}{cc}\frac{N P^{2} E_{0}}{j \omega Z_{C}} & \pm \frac{-N^{\prime}\left(\pi \cdot a_{0_{\text {avg }}}^{2}\right) \mu_{h} P H_{0}}{Z_{C}} \\ \pm \frac{\left(\pi \cdot a_{\text {mean }}^{2}\right) \mu_{h} N P E_{0}}{Z_{C}} & \frac{(-j \omega) N^{\prime}\left(\pi \cdot a_{0_{\text {avg }}^{2}}\right)^{2} \mu_{h}^{2} H_{0}}{Z_{C}}\end{array}\right]$

The polarizability coefficients are then obtained by scaling the dipole moments by the impressed field intensity:

$$
\left[\begin{array}{cc}
\alpha_{e e z} & \alpha_{e m z} \\
\alpha_{m e z} & \alpha_{m m z}
\end{array}\right]=\left\lfloor\begin{array}{cc}
\frac{p_{e e}}{E_{0}} & \frac{p_{e m}}{H_{0}} \\
\frac{p_{m e}}{E_{0}} & \frac{p_{m m}}{H_{0}}
\end{array}\right\rfloor
$$

\subsection{Conical coil inclusion characteristic impedance}

For a conical-coil we will represent the total coil impedance as $Z_{C}$, which is expressed in terms of a series of impedance components within the Bahr \& Clausing model as follows [7]:

$$
Z_{C}=R_{d c}+Z_{m c}+Z_{l c}+R_{w c}
$$

The energy lost due to dissipation of near, non-radiating, fields is expressed by the term $R_{d c}$. The second term estimates the average mutual impedance $Z_{m c}$ arising due to the nearest neighboring inclusions using the concept of the superposition of elemental dipoles and loops. The third term in $Z_{C}$ is the circuit impedance $Z_{l c}$ which is determined by modeling the inclusion as a perfect electrically conducting (PEC) center-fed electrically-small wire antenna embedded within the host medium and evaluated using the Method of Moments (MoM). Evaluation of $Z_{l c}$ in this manner will include a measure of the antenna radiation resistance as well. This differs from [7] wherein $Z_{l c}$ is evaluated for the 
center-fed helical coil modeled as two open-circuited transmission lines and the radiation resistance is calculated as a separate term in the total coil impedance. The surface resistivity of the inclusion wire material gives rise to the term $R_{w c}$. We present the expressions for each of the terms describing the total conical coil impedance $Z_{C}$, except for the transmission line contribution $Z_{l c}$ which is obtained using the Method of Moments [8].

In [7] the authors use the assumption that the dissipation arises in the helical coil due only to power lost through the ends of the wire, with the resulting relation being only dependent upon the separation distance of the wire ends. Using these same assumptions we leave the expression presented in [7] changed only slightly to include the effect of the conical coil angle on the wire ends separation distance.

$$
R_{d c} \approx \frac{\varepsilon_{h}{ }^{\prime \prime}}{\omega\left|\varepsilon_{h}\right|^{2}} \frac{8}{9 \pi\left(N P / \cos \left(\theta_{c}\right)\right)} e^{-k_{h} " N P / \cos \left(\theta_{c}\right)}
$$

where $\varepsilon_{h}$ is the complex permittivity of the host medium, $\varepsilon_{h}$ is the imaginary part of the host permittivity, $k_{h}^{\prime \prime}$ is the imaginary part of the complex wave number for the wave propagating in the surrounding host medium, and $\theta_{c}$ is the conical coil angle.

For the conical coil mutual impedance we assume this problem is separable into independent dipole and loop contributions and combine results to arrive at an expression of the total conical coil mutual impedance term [8]. $S$ is the inclusion separation distance, determined from the inclusion density, and $\beta$ is the propagation constant.

$$
Z_{m c} \approx 2 j \omega \mu \frac{e^{-j \beta S}}{S}\left\{\frac{(N P)^{2}}{\pi^{2}}+\beta^{2}\left(N^{\prime} a_{0_{\text {avg }}}^{2}\right)^{2}\right\}
$$

The component of inclusion resistance that relates to the wire resistivity is given by the following expression:

$$
R_{w c}=\frac{R_{s}}{\pi b} \cdot d_{w}
$$

where $R_{s}$ is the surface resistivity of the wire per unit length, $b$ is the diameter of the wire and $d_{w}$ is the total wire length.

\subsection{Circuit impedance of conical coil inclusion using Method of Moments}

From [7] we know that the helical coil may be well represented by a center-fed transmission line open-circuited at both ends. We have attempted to generalize the approach in [7] for use with a conical coil by evaluating the circuit impedance using the Method of Moments. The feed point of the structure is a delta-gap voltage source located at $1 / 2$ the height of the coil (Fig. 2). As dissipation due to wire resistance is estimated using (13) the wire in the MoM simulations is modeled as a perfect electric conductor (PEC). Our implementation of the MoM is based very closely upon the presentation provided in [9] and was developed by the authors in Matlab. By retaining only the first two terms of a Maclaurin series expansion of the Green's function in a MoM representation, it is assumed that the wire is electrically thin relative to the wavelength, this is the "thin-wire" approximation. One aspect of this approximation is that radial current contributions in each wire segment are ignored [9].

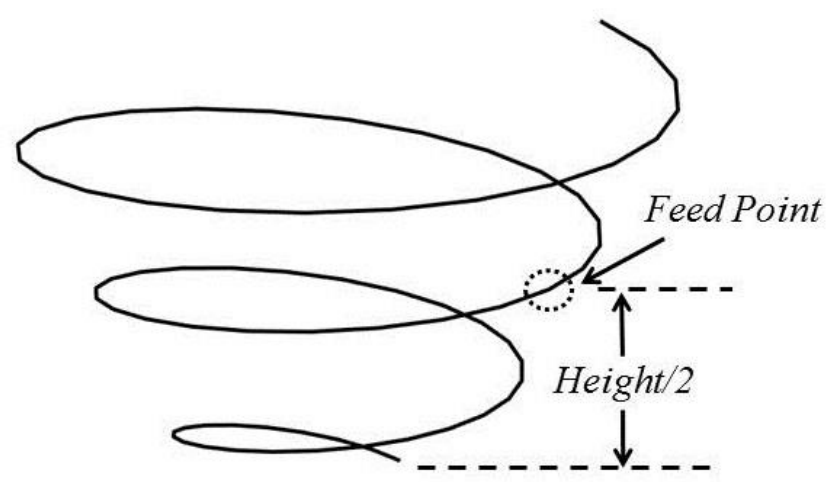

Figure 2: 3-Turn Conical Coil Illustrating Impedance Evaluation as Equivalent Electrically Small Antenna

\subsection{Material modeling using mixing relations}

The goal is the estimation of the macroscopic material parameters via implementation of mixing relations. The mixing relations may be thought of as the approximations employed to bridge the microscopic inclusion response to the macroscopic material response. Discussions of the derivation of mixing relations for chiral media, and corresponding limits of validity, are found in $[10,11,12]$. The polarizability relations are expressed in terms of the electric and magnetic dipole moments using the previous relations (10) and (11) and the inclusion density $n[1,7]$.

$$
\kappa=j \frac{n \alpha_{e m}}{2 \sqrt{\mu_{h} \varepsilon_{h}} \Delta}
$$

$$
\begin{aligned}
& \mu=\mu_{h}+\frac{n}{2 \Delta}\left\lfloor\alpha_{m m}-\frac{n}{6 \varepsilon_{h}}\left(\alpha_{e e} \alpha_{m m}-\alpha_{e m} \alpha_{m e}\right)\right\rfloor \\
& \varepsilon=\varepsilon_{h}+\frac{n}{2 \Delta}\left\lfloor\alpha_{e e}-\frac{n}{6 \mu_{h}}\left(\alpha_{e e} \alpha_{m m}-\alpha_{e m} \alpha_{m e}\right)\right\rfloor
\end{aligned}
$$


where

$$
\Delta=1-\frac{n}{6}\left\lfloor\frac{\alpha_{e e}}{\varepsilon_{h}}+\frac{\alpha_{m m}}{\mu_{h}}-\frac{n}{6 \mu_{h} \varepsilon_{h}}\left(\alpha_{e e} \alpha_{m m}-\alpha_{e m} \alpha_{m e}\right)\right\rfloor
$$

\subsection{Electromagnetic response of Pasteur slab}

Consider a slab of Pasteur material (Region 2) sandwiched between two simple isotropic spaces (Regions 1 and 3) as pictured in Fig. 3 [1]. For this work we assume Regions 1 and 3 are free-space $[1,8]$. In [7] it is demonstrated that the inverse circular polarization ratio, $q$, gives us the ratio of the left-handed polarized (LHP) and right-handed polarized (RHP) waves at the exit of a chiral slab.

$$
q=\exp \left(j\left(\psi_{L H}-\psi_{R H}\right) / 2\right) \cdot \frac{1-R^{2} \exp \left(j \psi_{R H}\right)}{1-R^{2} \exp \left(j \psi_{L H}\right)} \equiv|q| e^{j \theta_{q}}
$$

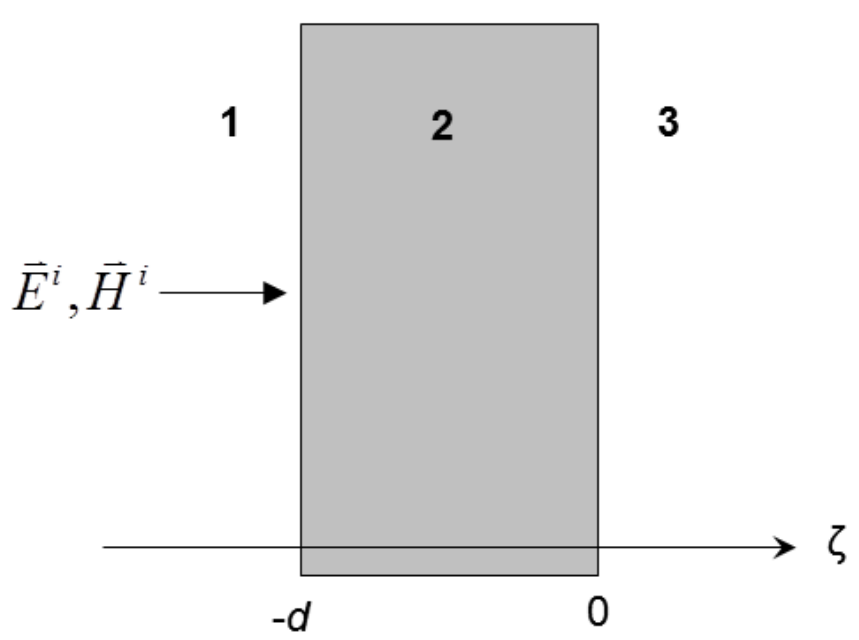

Figure 3: Pasteur Slab Sandwiched Between Two Isotropic Half-Spaces [1]

The LHP and RHP propagation constants $\psi_{L H}$ and $\psi_{R H}$ are expressed as follows: where $d$ is the slab thickness, $\mu_{h}$ and $\varepsilon_{h}$ are the host permeability and permittivity, $\mu_{r}$ and $\varepsilon_{r}$ are the chiral material permeability and permittivity relative to the host medium, $\omega$ is the radian frequency, and $\kappa$ the chirality parameter [7].

$$
\psi_{\left(\begin{array}{l}
L H \\
R H
\end{array}\right)}=-2 \omega \sqrt{\mu_{h} \varepsilon_{h}}\left(\sqrt{\mu_{r} \varepsilon_{r}} \mp \kappa\right) d
$$

The factor $R$ is the following ratio of chiral wave impedance, $\eta_{c}$, to the wave impedance of the medium external to the slab, $\eta_{0}$, which is defined as free-space in this example.

$$
R=\frac{\eta_{c}-\eta_{o}}{\eta_{c}+\eta_{o}}
$$

The tilt angle, $\tau$, of the elliptical wave at the exit surface of the slab may be determined as half of the angle, $\theta_{q}$, which is the phase angle of the complex representation of $q$ in (20) [7].

$$
\tau=\frac{1}{2} \theta_{q}
$$

The axial ratio, $A R$, may also be expressed in terms of the inverse circular polarization ratio [7]:

$$
A R=\frac{|1+| q||}{|1-| q||}
$$

\section{Results: Pasteur media using conical coil inclusions}

The input impedance minima of the transmissionline/antenna representation of a chiral wire coil inclusion defines the regions of large chirality, and hence polarization conversion [7]. We begin by examining the simulated complex input impedance of a conical coil versus conical angle with the coil possessing the following dimensions: minimum coil radius of $a_{0}=0.625 \mathrm{~mm}$, helix pitch of $P=0.667 \mathrm{~mm}$, wire diameter of $b=0.1524 \mathrm{~mm}$, number of turns $N=3$, Copper Wire immersed in a host material of Dow Corning 3110 RTV rubber $\left(e_{r}=2.95-\mathrm{j} 0.07\right)$ with and inclusion density of $n=10 \cdot 10^{6} \mathrm{~m}-3[7,8]$.

\subsection{Circuit impedance of the conical coil inclusion versus conical angle}

In the following figure we overlay the magnitudes of the conical coil antenna input impedance $\left(Z_{l c}\right)$ as determined using the Method-of-Moments. The conical angles examined are: $0^{\circ}, 5^{\circ}, 15^{\circ}$ and $30^{\circ}$.

For the 3-turn helical coil, with a $0^{\circ}$ conical angle, we note that the location of the 2 nd impedance minimum is at nearly three times the frequency of the fundamental resonance near $21 \mathrm{GHz}$, and with the impedance maximum occurring at approximately twice the fundamental resonance frequency. However, as the conical angle increases additional impedance maxima and minima appear over the observed frequency bandwidth.

For a conical angle of $30^{\circ}$ we find the fundamental resonance, which is determined primarily by the overall wire length, has shifted downward from just over $7 \mathrm{GHz}$ to nearly $3 \mathrm{GHz}$ and the relative relationship of the impedance maxima and minima has changed dramatically when 
compared to that for a helical coil. For the $30^{\circ}$ coil we now find that the 2 nd impedance minimum is closer to twice the fundamental resonance frequency. The introduction of the conical angle has created this new impedance resonance near twice the fundamental that is not present in the $0^{\circ}$ helical coil. The asymmetry of the conical coil, when viewed as a center-fed transmission line, provides the additional resonance formation.

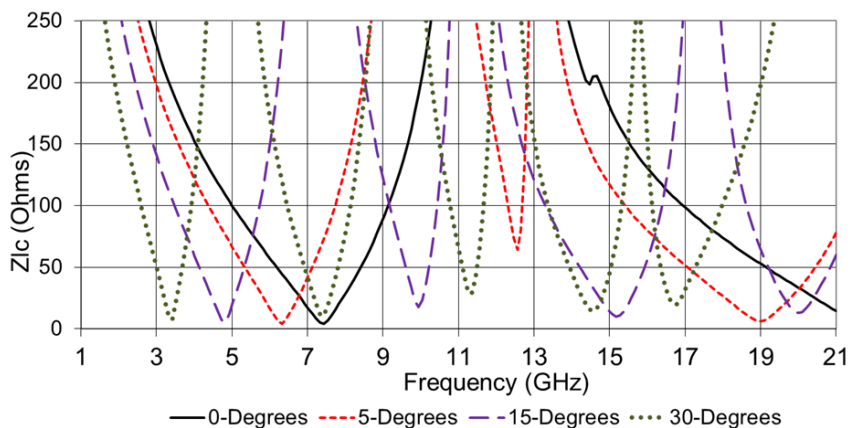

Figure 4: Magnitude of Conical Coil $\mathrm{Z}_{\mathrm{lc}}$ Complex Circuit Impedance at $0^{\circ}, 5^{\circ}, 15^{\circ}$, and $30^{\circ}$ Conical Angles.

It is important to note that only the transmission line impedance contribution, $Z_{l c}$, results in resonance phenomena. However, the other impedance contributions of of dissipation resistance $\left(R_{d}\right)$, surface resistivity of the inclusion wire material $\left(R_{w}\right)$, and mutual impedance $\left(Z_{m}\right)$ all serve to modify the total conical coil impedance significantly, with the overall result being an increasing coil impedance with frequency.

\subsection{Material parameters of dielectric slab loaded with conical coil inclusions}

When using mixing relations to model effective material parameters it is assumed that the electric field remains uniform throughout the volume of the inclusion, requiring that the inclusion be electrically small. For a 3-turn $30^{\circ}$ conical coil inclusion of dimensions specified previously in this report, the volume enveloping the inclusion is approximately $42 \cdot 10^{-9} \mathrm{~m}^{3}$ (estimating sphere radius as approximately half the coil height). The Rayleigh requirement for valid use of the mixing relations is $k_{\text {eff }} D<1$

[1]; where $k_{\text {eff }}$ is the effective wavenumber in the chiral medium, and $D$ is the diameter of an imaginary sphere enclosing the inclusion. The highest frequency to be modeled using mixing relations for this inclusion, embedded within a host material of dielectric constant of $2.95-\mathrm{j} 0.07$, is approximately $14 \mathrm{GHz}$. Our material results are plotted up to $10 \mathrm{GHz}$ to stay well below this frequency limit.

In the following figure the complex material parameters for a dielectric slab loaded with conical coil wire inclusions is presented. The inclusions are of the same design as presented previously and are repeated here: minimum coil radius of $a_{0}=0.625 \mathrm{~mm}$, helix pitch of $P=0.667 \mathrm{~mm}$, wire diameter of $b=0.1524 \mathrm{~mm}$, number of turns $N=3$, conical angle of $\theta_{c}=30^{\circ}$, with an inclusion density of $\mathrm{n}=10 \cdot 10^{6} \mathrm{~m}^{-3}$, immersed in a host material of Dow Corning 3110 RTV rubber $\left(e_{r}=2.95-\mathrm{j} 0.07\right)$. This inclusion design is the coil geometry from [7] modified by a $30^{\circ}$ conical angle. Note that the first two material resonances are found to lie within the Rayleigh criterion, so application of mixing relations is valid for estimating material parameters at these frequencies.
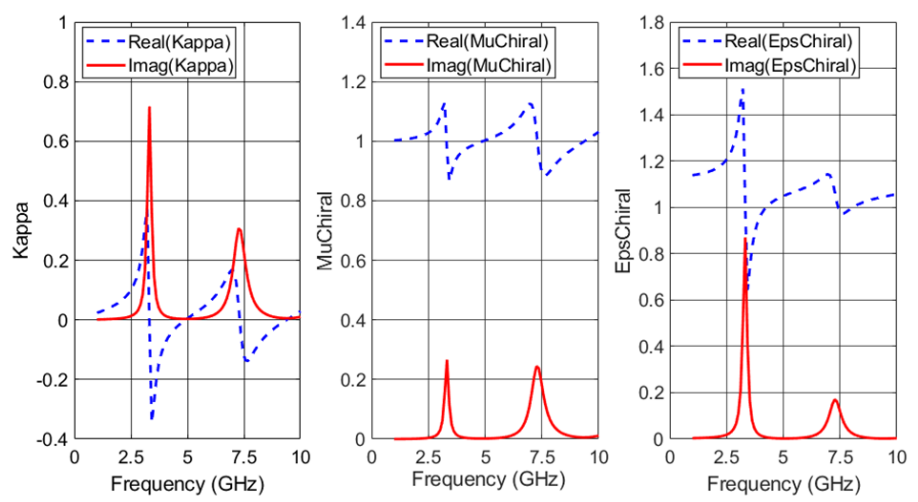

Figure 5: Complex Normalized Relative Material Parameters for Host Loaded with 3-Turn $30^{\circ}$ Conical Coils.

In Fig. 5 we see that both the real and imaginary parts of the effective normalized relative material permittivity (EpsChiral) and permeability (MuChiral) increase in magnitude for increasing complex chirality. These effects are especially pronounced around the chiral resonances which, in turn, correspond well in frequency with the impedance minima for each coil as shown previously. Such deviations in permittivity and permeability have been used by researchers to design absorbing or polarization converting materials by carefully dimensioning inclusion and material parameters $[1,7]$.

\subsection{Axial ratio response of dielectric slab loaded with conical coil inclusions}

To demonstrate tuning of the conical coil fundamental resonance, and utility of a higher-order resonance, we present the predicted transmitted axial ratio (AR) for a dielectric slab loaded with conical coil wire inclusions illuminated with linearly polarized electromagnetic radiation. The dimensions of the inclusions, inclusion density, and chosen host medium, were specified previously in this paper. The slab thickness was set at $1.9 \mathrm{~cm}$ as this resulted in AR values closest to unity for the first two coil resonances, determined via trial-and-error.

Results obtained using our method are compared to results determined using the tumble-averaged-forwardscattering (TAFS) approach [13]. The conical coil was discretized at the same resolution for both techniques. In the TAFS approach 500 random orientations of the inclusion were produced and the complex co-polarized and cross- 
polarized far-fields calculated for two separate incident orthogonal linearly polarized plane waves [13]. These averaged far-field results were used to estimate the polarization conversion at the output of the $1.9 \mathrm{~cm}$ thick slab.

The results in Fig. 6 compare the calculated AR for the approach developed in this effort versus the TAFS method. General agreement between the approximate frequency locations of the two material resonances is displayed. The TAFS resonance predictions are shifted higher in frequency than the mixing relation results. The difference in frequency locations of AR minima between the two approaches was found to be generally independent of the coil discretization. The higher frequency resonance is not as pronounced in terms of polarization conversion and bandwidth for the TAFS prediction as compared to the $\mathrm{MoM} /$ mixing-relation prediction.

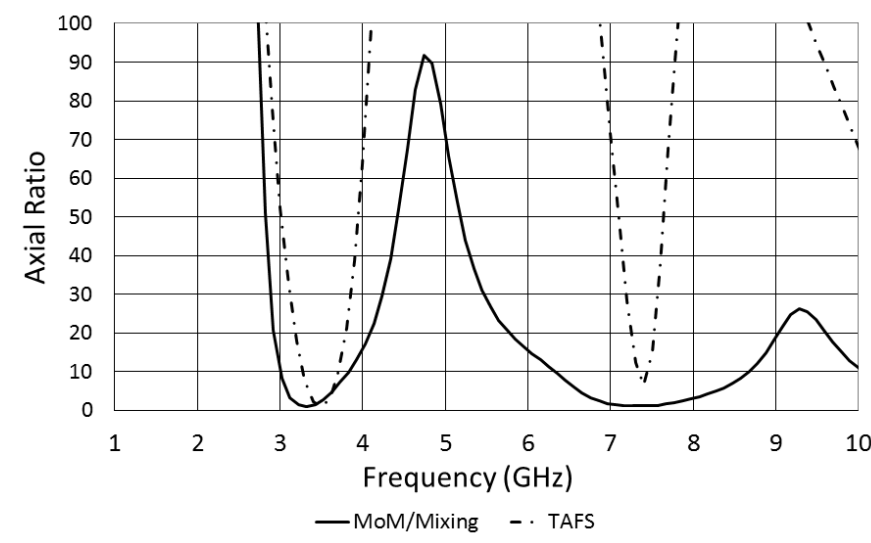

Figure 6: Predicted Axial Ratio of $1.9 \mathrm{~cm}$ Thick Dielectric Slab Loaded with 3-Turn $30^{\circ}$ Conical Coil Inclusions.

One drawback of TAFS is that the approach ignores inclusion interactions as it is based solely upon the calculated far-field scattering for a single inclusion averaged over many randomly selected orientations [13]. The TAFS approach is also extremely time consuming requiring nearly 5 days of processing when run on a modest Dell M4800 quad-core laptop computer with $8 \mathrm{~GB}$ RAM. This is to be compared to the approach developed in this effort which required only a few hours to complete. Some disagreement between the two approaches is expected as parameters derived via scattering of a plane wave by a single conical coil is not completely analogous to the mixing relation approach.

Future work may include use of available commercial CEM software (e.g. Ansys HFSS) for refining the approach presented in this report. The creation of a set of chiral slabs using commercially available small multi-turn conical coils is also being investigated.

\section{Conclusions}

In this work we have investigated the electromagnetic properties of the multi-turn conical wire coil as a chiral inclusion within a dielectric host medium. We have provided expressions for the various impedance components of the multi-turn conical wire coil via extension of an existing approach for helical coils [7]. The conical coil wire inclusion was found to demonstrate the ability of frequency tuning of impedance minima through variation of conical angle. Frequencies corresponding to chiral resonances, and also maximum variation of effective material parameters, can be designed by appropriate choice of coil dimensions including the conical angle.

\section{Acknowledgements}

The authors would like to thank Dr. Andrew Gatesman for providing helpful suggestions.

\section{References}

[1] I.V. Lindell, A.H. Sihvola, S.A. Tretyakov, A.J. Viitanen, Electromagnetic Waves in Chiral and Bi-Isotropic Media, Norwood, MA, Artech House, Inc., 1994.

[2] D.K. Kalluri, Electromagnetics of Time Varying Complex Media: Frequency and Polarization Transformer- $2^{\text {nd }}$ Edition, Taylor Francis, CRC Press, April 2010.

[3] R. Marques, F. Martin, Metamaterials with Negative Parameters: Theory, Design and Microwave Applications. Wiley Press - Interscience, 2013.

[4] A. Taflove, Computational Electrodynamics: The Finite Difference Time Domain Method, Norwood, MA, Artech House, 1995.

[5] T.J. Cui, D. Smith, R. Liu, Metamaterials: Theory, Design and Applications, Springer-Verlag Publishing, 2010

[6] A. Priou, A. Sihvola, S. Tretyakov, A. Vinogradov, Advances in Complex Electromagnetic Materials, Springer Science and Business Media, December 2012.

[7] A.J. Bahr and K.R. Clausing, "An Approximate Model for Artifical Chiral Material”, IEEE Transactions on Antennas and Propagation, Vol. 42, No. 12, Dec.,, pp. 1592-1599, 1994.

[8] G.B. DeMartinis, "Chiral Media Using Conical Coil Inclusions", D.Eng. Thesis, Department of Electrical and Computer Engineering, University of Massachusetts Lowell, Lowell, MA, 2008.

[9] R.F. Harrington, Field Computation by Moment Methods, Wiley-IEEE Press, May 1993.

[10] A.H. Sihvola and I.V. Lindell, "Chiral Maxwell-Garnett Mixing Formula", IEEE Electronics Letters, Vol. 26, No. 2, Jan., pp. 118-119, 1990.

[11] A.H. Sihvola, "Bi-Isotropic Mixtures", IEEE Transactions on Antennas and Propagation, Vol. 40, No. 2, Feb., pp. 188-197, 1990.

[12] A.H. Sihvola, "How Strict are Theoretical Bounds for Dielectric Properties of Mixtures?", IEEE Transactions on Geoscience and Remote Sensing, Vol. 40, No. 4, pp. 880- 886, 2002.

[13] R. Luebbers, H.S. Langdon, F. Hunsberger, C.F. Bohren, and S. Yoshikawa, "Calculation and Measurement of the Effective Chirality Parameter of a Composite Material Over a Wide Frequency Band", IEEE Transactions on Antennas and Propagation, Vol. 43, No. 2, Feb., pp. 123-130, 1995. 